\title{
The effect of direct interactions on Brownian diffusion
}

\author{
C. Van den Broeck, ${ }^{\text {a) }}$ F. Lostak, and H. N. W. Lekkerkerker \\ Vrije Universiteit Brussel, Fakulteit Wetenschappen, Brussels, Belgium \\ (Received 14 November 1979; accepted 31 January 1980)
}

\begin{abstract}
The effect of direct interactions between suspended particles on their diffusion coefficient is investigated starting from the generalized Einstein relation. It is shown that an attractive potential added to the hard core repulsion leads to a decrease of the diffusion coefficient, whereas a repulsive term has the opposite effect. Simple examples of attractive and repulsive potentials are considered in some detail. Using these results the possibility to obtain information on the interaction potential between suspended particles from their diffusion coefficient is discussed.
\end{abstract}

\section{INTRODUCTION}

The study of the diffusion of interacting particles has recently been the object of much theoretical ${ }^{1-14}$ and experimental ${ }^{15-22}$ work. The quantitative interpretation of the experimental data has been confused by the fact that even for the simple case of hard spheres a number of different theoretical results have been given.

Basically two different theoretical approaches to the problem can be distinguished. The first approach ${ }^{1-12}$ starts from a Smoluchowski equation for the $N$-particle probability distribution in configuration space. This description must then be reduced to a closed equation for the one-body distribution function which displays the diffusion coefficient explicitly. The second, ${ }^{13}$ much simpler, approach is based upon an extension of the Einstein argument, ${ }^{23,24}$ relating mobility and diffusion, to the case of interacting particles. The problem that then remains is the calculation of the density dependence of the mobility and the osmotic compressibility. Recently, Felderhof ${ }^{14}$ has shown by explicit calculation that the results obtained from the Smoluchowski equation are in complete agreement with the ones obtained by Batchelor from the generalized Einstein approach. Felderhof also indicated why previous authors failed to find this agreement.

In this paper we discuss the influence of static interactions between the suspended particles on their diffusion coefficient starting from the generalized Einstein relation. We first give a simple derivation of this fundamental relation and present the density dependence of the mobility and the osmotic compressibility. These results then allow us to consider the density dependence of the diffusion coefficient for a given interaction potential between the particles. General conclusions are drawn for the case that the potential comprises in addition to a hard core part a (purely) attractive or (purely) repulsive part. Specific examples of such potentials are considered in some detail. Based upon these results, we discuss the possibility to obtain information on the interaction potential between suspended particles from the density dependence of their diffusion coefficient.

\footnotetext{
a)Aspirant N.F.W.O. Belgium.
}

\section{DIFFUSION MOBILITY AND OSMOTIC COMPRESSIBILITY}

Consider a system of suspended particles with number density $n$. Local differences of this density will be smoothed out by the process of diffusion. This tendency can be described by a current density $\mathbf{j}$ which is proportional to $\operatorname{grad} n$,

$$
\mathbf{j}=-D \operatorname{grad} n \text {. }
$$

Einstein ${ }^{23,24}$ has shown that the diffusion coefficient $D$ in Eq. (1) can be written

$$
D=b\left(\frac{\partial \Pi}{\partial n}\right)_{T, P},
$$

where $b$ is the mobility of the suspended particles and $\Pi$ is their osmotic pressure. Since Eq. (2) is of fundamental importance in the further discussion we briefly sketch its derivation following Einstein's argument. Consider a cylindrical vessel filled with a suspension in thermal and mechanical equilibrium, in which diffusion of particles takes place as the result of a concentration gradient along the (horizontal) $Z$ axis (see Fig. 1). The thermodynamic driving force for this diffusion process is the osmotic pressure gradient along the $Z$ axis. Thus, for instance, the suspended particles enclosed between the vertical sections $S$ and $S^{\prime}$ of the vessel at the positions $z$ and $z+d z$ are subject to a force in the $Z$ direction given by

$$
[\Pi(z)-\Pi(z+d z)] A,
$$

where $A$ is the area of the cross section of the vessel. Thus, we obtain for the thermodynamic force per suspended particle measured along the $Z$ axis

$$
f=-\frac{1}{n} \frac{\partial \Pi}{\partial z}=-\frac{1}{n}\left(\frac{\partial \Pi}{\partial n}\right)_{T, P} \frac{\partial n}{\partial z} .
$$

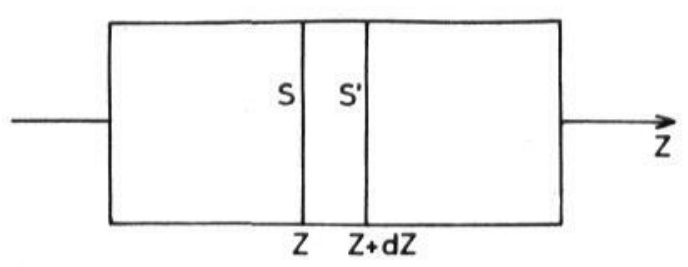

FIG. 1. Diffusion of Brownian particles in a cylindrical vessel under influence of an osmotic pressure gradient along the horizontal $Z$ axis. 
As a result of this force the particles acquire an average velocity with respect to a volume fixed frame of reference

$$
\langle U\rangle=b f,
$$

where $b$ is the mobility. The resulting current density $j$ thus reads

$$
j=n\langle U\rangle=-b\left(\frac{\partial \Pi}{\partial n}\right)_{T, P} \frac{\partial n}{\partial z} .
$$

Comparing this result with expression (1) for the current density one is led to the Einstein relation (2).

Note that in the derivation the thermodynamic force is treated as if it were an external force acting on the suspended particles, whereas it is clear that its origin is of a statistical nature. Therefore, Eq. (4) is to be understood as a thermodynamic result valid on macroscopic time and length scales. On such scales Onsager's assumption ${ }^{25}$ is valid and states that a system cannot distinguish between external and thermodynamic forces. It should also be noted that, since $b$ represents the mobility with respect to a volume fixed frame of reference, Eq. (2) is an expression for the diffusion coefficient in this same frame of reference.

At infinite dilution where all interactions between the particles can be neglected the osmotic pressure is given by the Van't Hoff equation

$$
\Pi=\Pi_{0}=n k T,
$$

and for spherical particles the mobility reduces to the Stokes expression

$$
b=b_{0}=1 / 6 \pi \eta a .
$$

Here $a$ is the hydrodynamic radius of the suspended particles and $\eta$ is the shear viscosity of the suspending medium. Substituting the above expressions in Eq. (2) one obtains the famous Stokes-Einstein expression for the diffusion coefficient

$$
D_{0}=k T / 6 \pi \eta a \text {. }
$$

In general, however, there will be both static and hydrodynamic interactions between the particles affecting the osmotic pressure and the mobility, respectively. At low concentrations these effects can be taken into account by expanding $\Pi$ and $b$ in a power series of the concentration

$$
\begin{aligned}
& \Pi=k T\left(n+B_{2} n^{2}+\cdots\right), \\
& b=(1 / 6 \pi \eta a)(1+\lambda \phi+\cdots) .
\end{aligned}
$$

Here $B_{2}$ is the second osmotic virial coefficient and $\phi$ is the volume fraction

$$
\phi=\frac{4}{3} \pi a^{3} n \text {. }
$$

The results given in Eqs. (6) to (8) together with Eq.

(2) allow one to write the diffusion coefficient as

$$
D=D_{0}\left(1+k_{D} \phi+\cdots\right) .
$$

Here

$$
k_{D}=C+\lambda,
$$

with

$$
C=2 B_{2} / \frac{4}{3} \pi a^{3}
$$

The coefficient $C$ can be obtained from the MacMillanMayer $^{26,27}$ virial expansion for the osmotic pressure

$$
C=-\frac{3}{a^{3}} \int_{0}^{\infty}\left[\exp \left(\frac{-W_{12}}{k T}\right)-1\right] r^{2} d r
$$

where $W_{12}(r)$ is the potential of mean force between the suspended particles. The density dependence of the mobility was first considered by Burgers ${ }^{28}$ and further refined by Batchelor. ${ }^{29}$ From the latter work one obtains

$$
\begin{aligned}
\lambda=\frac{3}{a^{3}} \int_{0}^{a}[ & \left.\exp \left(\frac{-W_{12}}{k T}\right)-1\right] r^{2} d r+\frac{3}{a^{2}} \int_{a}^{\infty}\left[\exp \left(\frac{-W_{12}}{k T}\right)-1\right] \\
& \times r d r+\frac{1}{2}+\frac{1}{a^{3}} \int_{2 a}^{\infty} \exp \left(\frac{-W_{12}}{k T}\right) \\
& \times\left[A(r)+2 B(r)-3\left(1+\frac{a}{r}\right)\right] r^{2} d r
\end{aligned}
$$

The first three terms represent the effect of the motion of the surrounding particles on the particle under consideration. The last term takes into account the effect of the motion of the particle under consideration induced in itself by the presence of the other particles. In Eq. (13), $b_{0} A(r)$ is the mobility of a pair of suspended particles separated by a distance $r$ along the line connecting their centers and $b_{0} B(r)$ is the mobility perpendicular to this line. Exact expressions for $A(r)$ and $B(r)$ have been obtained by Stimson and Jeffery ${ }^{30}$ and Goldman, Cox, and Brenner, ${ }^{31}$ respectively.

In deriving Eq. (13), the direct nonhydrodynamical interactions have been accounted for in a statistical sense in that they affect the pair correlation function. The effect on the dynamics itself, calculated for the case of one particle moving in a thermal ensemble of surrounding particles by Phillies, ${ }^{32}$ will not be considered here.

The results given in Eqs. (12) and (13) allow one to calculate the density dependence of the diffusion coefficient for a given potential.

\section{EFFECTS OF DIRECT INTERACTIONS}

\section{A. General considerations}

In the discussion of the effect of direct interactions it is convenient to distinguish the contribution of the hard core from the remaining interactions. This is achieved by writing

$$
W_{12}=W_{12}^{H S}+W_{12}^{I N T},
$$

where

$$
W_{12}^{\mathrm{HS}}=\left\{\begin{array}{l}
\infty \text { for } 0 \leq r \leq 2 a, \\
0 \text { for } r>2 a .
\end{array}\right.
$$

Substituting Eq. (14) in (12), one obtains

$$
C=C^{\mathrm{HS}}+C^{\mathrm{INT}} \text {, }
$$

where

$$
C^{\mathrm{HS}}=8
$$

and 


$$
C^{\mathrm{INT}}=-\frac{3}{a^{3}} \int_{2 a}^{\infty}\left[\exp \left(\frac{-W_{12}}{k T}\right)-1\right] r^{2} d r .
$$

From Eq. (17) it is clear that an attractive $W_{12}^{\text {INT }}$ leads to decrease of the osmotic compressibility whereas a repulsive $W_{12}^{\text {INT }}$ has the opposite effect. Similarly one obtains for the mobility

$$
\lambda=\lambda^{\mathrm{HS}}+\lambda^{\mathrm{INT}},
$$

where

$$
\lambda^{\mathrm{HS}}=-6.55
$$

and

$$
\begin{aligned}
\lambda^{\mathrm{INT}}= & \frac{3}{a^{2}} \int_{2 a}^{\infty}\left[\exp \left(\frac{-W_{12}}{k T}\right)-1\right] r d r \\
& +\frac{1}{a^{3}} \int_{2 a}^{\infty}\left[\exp \left(\frac{-W_{12}}{k T}\right)-1\right] \\
& \times\left[A(r)+2 B(r)-3\left(1+\frac{a}{r}\right)\right] r^{2} d r \\
= & \frac{1}{a^{3}} \int_{2 a}^{\infty}\left[\exp \left(\frac{-W_{12}}{k T}\right)-1\right] \\
& \times[A(r)+2 B(r)-3] r^{2} d r .
\end{aligned}
$$

The result (19) was first obtained by Batchelor ${ }^{13}$ using numerical values for $A(r)$ and $B(r)$ derived from the exact expressions for these quantities. From these values it also follows that $[A(r)+2 B(r)-3]$ is always positive. Thus an attractive $W_{12}^{\mathrm{INT}}$ leads to an increase of the mobility whereas a repulsive $W_{12}^{\text {INT }}$ has the opposite effect. Physically this can be understood from the fact that a pair of particles moving close to each other drag one another, whereas in a volume fixed frame of reference the effect of the backflow hinders the motion of particles far away from one another. Combining Eqs. (10) and (15)-(20), one obtains

$$
k_{D}=k_{D}^{\mathrm{HS}}+k_{D}^{\mathrm{INT}},
$$

where

$$
k_{D}^{\mathrm{Hs}}=1.45
$$

and

$$
\begin{aligned}
k_{D}^{\mathrm{INT}}= & +\frac{1}{a^{3}} \int_{2 a}^{\infty}\left[\exp \left(\frac{-W_{12}}{k T}\right)-1\right] \\
& \times[A(r)+2 B(r)-6] r^{2} d r .
\end{aligned}
$$

From the values for $A$ and $B$ compiled by Batchelor it follows that $[A(r)+2 B(r)-6]$ is always negative. This implies that a purely attractive $W_{12}^{\text {INT }}$ leads to a decrease of the diffusion coefficient whereas a purely repulsive $W_{12}^{1 N T}$ has the opposite effect. Thus we find that the diffusion coefficient follows the behavior of the osmotic compressibility. In fact, it can even be shown that in these cases:

$$
\left|\lambda^{\mathrm{INT}}\right| \leq \frac{1}{2}\left|C^{\mathrm{INT}}\right| \text {. }
$$

This inequality is based upon the fact that

$$
A(r)+2 B(r)-3 \leq 3 a / r \quad(r \geq 2 a),
$$

which follows again from the values tabulated by Batchelor. Substituting Eq. (25) in Eq. (20b), it immediately follows that

$$
\left|\lambda^{\mathrm{INT}}\right| \leq \frac{3}{a^{2}} \int_{2 a}^{\infty}\left|\exp \left(\frac{-W_{12}}{k T}\right)-1\right| r d r .
$$

Thus, we clearly have

$$
\begin{aligned}
\left|\lambda^{\mathrm{INT}}\right| & \leq \frac{3}{a^{2}} \int_{2 a}^{\infty}\left|\exp \left(\frac{-W_{12}}{k T}\right)-1\right| r \cdot \frac{r}{2 a} d r \\
& =\frac{1}{2}\left|C^{\mathrm{INT}}\right|,
\end{aligned}
$$

which completes the proof of Eq. (24). One notes that no similar general conclusions can be obtained for potentials with both attractive and repulsive contributions in $W_{12}^{\mathrm{INT}}$.

\section{B. Attractive and repulsive potentials}

A very simple model to take into account the effect of attractive forces has been proposed by Batchelor. ${ }^{29}$ In this model, the effect of attractive forces is simulated by an excess number $\alpha \phi$ of nearly touching particles. This means that the pair correlation function to lowest order in the density $g_{(0)}(r)$ is given by

$$
g_{(0)}(r)=g_{(0)}^{\mathrm{HS}}(r)+\frac{1}{12} \alpha a \delta(r-2 a) .
$$

Substitution of $g_{(0)}=\exp \left(-W_{12} / k T\right)$ in Eqs. (7), (20a), and (23) yields

$$
\begin{aligned}
& C^{\mathrm{INT}}=-\alpha, \\
& \lambda^{\mathrm{INT}}=0.5 \alpha-0.06 \alpha=0.44 \alpha, \\
& k_{D}^{\mathrm{INT}}=-0.56 \alpha .
\end{aligned}
$$

In Eq. (27b) we have written explicitly the contribution $0.5 \alpha$ arising from the direct hydrodynamic effect of surrounding particles on the particles under consideration, and the induction contribution $-0.06 \alpha$. Note that the latter contribution is small so that one may generally approximate $\lambda^{\mathrm{INT}}$ by only its direct contribution [compare with Eq. (20a)]

$$
\lambda^{\mathrm{INT}}=\frac{3}{a^{2}} \int_{2 a}^{\infty}\left[\exp \left(\frac{-W_{12}}{k T}\right)-1\right] r d r .
$$

This approximate expression of $\lambda^{\mathrm{INT}}$ allows considerable simplification in analytic calculations and we will henceforth use it. The error is maximal for short range potentials, such as the one considered here, and is then of the order of $10 \%$. From Eq. (27c) note that in agreement with the general conclusions reached in Sec. III. A, the diffusion coefficient decreases for increasing $\alpha$. In particular, for $\alpha \simeq 3$, the effect of the attractive interactions cancels the effect of the hard core and the Stokes-Einstein value $D_{0}$ for the diffusion coefficient is recovered.

A more detailed model for attractive interactions that still can be handled easily is the square well model

$$
W_{12}(r)= \begin{cases}\infty & \text { for } 0 \leq r \leq 2 a, \\ -y k T & \text { for } 2 a<r \leq 2 a(1+x), \\ 0 & \text { for } 2 a(1+x)<r .\end{cases}
$$

For this model potential the attractive contribution to the osmotic compressibility is of the form

$$
C^{\mathrm{INT}}=-8\left(e^{y}-1\right)\left[(1+x)^{3}-1\right] .
$$


From Eqs. (28) and (29), one obtains for $\lambda^{\mathrm{INT}}$,

$$
\lambda^{\mathrm{INT}}=6\left(e^{y}-1\right)\left[(1+x)^{2}-1\right] .
$$

Combining Eqs. (30) and (31), one finds for the coefficient $k_{D}^{\mathrm{INT}}$,

$$
k_{D}^{\mathrm{INT}}=-2\left(e^{y}-1\right)\left[(1+x)^{2}(1+4 x)-1\right] .
$$

Results (30)-(32) are in agreement with those obtained by Altenberger. ${ }^{4}$ However, the hard sphere contribution in the latter paper is accounted for incompletely. Note that $C^{\mathrm{INT}}$ and $\lambda^{\mathrm{INT}}$, and consequently $K_{D}^{\mathrm{INT}}$, have the same dependence on the well depth $y k T$. Thus the ratio of any two of these quantities depends only on the range of the potential $2 a x$. For short range potentials $x \ll 1$, the ratio $\left|\lambda^{\mathrm{INT}} / C^{\mathrm{INT}}\right|$ is close to its maximal value $\frac{1}{2}$ [compare with Eq. (24)]. For increasing range of the potential this ratio decreases and it thus gives an indication of the range of the potential.

As the counterpart of the simple $\alpha$ model for attractive interactions, we simulate the effect of repulsive interactions by introducing an effective hard sphere diameter $2 a(1+\beta)$ with $\beta>0$. One then obtains

$$
\begin{aligned}
& C^{\mathrm{INT}}=8\left[(1+\beta)^{3}-1\right], \\
& \lambda^{\mathrm{INT}}=-6\left[(1+\beta)^{2}-1\right],
\end{aligned}
$$

and thus

$$
k_{D}^{\mathrm{INT}}=+2\left[(1+\beta)^{2}(1+4 \beta)-1\right] .
$$

Note that the ratio $\left|\lambda^{\mathrm{INT}} / C^{\mathrm{INT}}\right|$ is the same here as for the square well potential.

As a more realistic example for a repulsive potential we consider a shielded Coulomb potential. More specifically we study the case of charged particles surrounded by a thin double layer, i.e., the Debye length $\xi$ is much smaller than the radius of the particle. The shielded Coulomb potential can then be written ${ }^{33}$

$$
W_{12}(r)=A k T \ln \{1+\exp [-(r-2 a) / \xi]\} \quad(r \geq 2 a),
$$

where $A>0$ is a strength factor which is related to the surface charge or surface potential of the particle. Substituting Eq. (36) in Eqs. (17) and (28), one obtains in principle the dependence of the diffusion coefficient on $A$ and $\xi$. However, it is possible to obtain convenient approximate analytic expressions by suitably exploiting the short range character of the repulsive interaction (36). Indeed the quantity

$$
\exp \left(-W_{12} / k T\right)-1=\left\{1+\exp \left[-\frac{(r-2 a)}{\xi}\right]\right\}^{-A}-1
$$

is only appreciably different from zero for $r$ close to 2a. This allows us to approximate Eqs. (17) and (28) by

$$
C^{\mathrm{INT}} \simeq-\frac{12}{a} \int_{2 a}^{\infty}\left[\exp \left(\frac{-W_{12}}{k T}\right)-1\right] d r
$$

and

$$
\lambda^{\mathrm{INT}} \simeq+\frac{6}{a} \int_{2 a}^{\infty}\left[\exp \left(\frac{-W_{12}}{k T}\right)-1\right] d r .
$$

The remaining quantity

$$
\delta=-\int_{2 a}^{\infty}\left[\exp \left(\frac{-W_{12}}{k T}\right)-1\right] d r
$$

can be effectively interpreted as an increase of the hard core diameter from $2 a$ to $2 a+\delta$. When $A$ is large the value of $\delta$ can be approximately written (see Appendix)

$$
\delta \simeq \xi(\ln A+C),
$$

where $C$ denotes Euler's constant

$$
C=0.5772 \cdots \text {. }
$$

Expression (40) can be given a simple physical meaning by noting that

$$
W_{12}(2 a+\delta) \simeq k T e^{-c}=0.561 k T .
$$

Thus the effective diameter equals the actual diameter increased by the distance $\delta$ for which the potential has decreased to the value $e^{-c} k T$. A similar interpretation has been given by Onsager ${ }^{34}$ for the osmotic pressure for the case of Coulomb interaction between two plane double layers. Finally, conclude from Eq. (40) that $\delta$ may be significantly larger than $\xi$.

\section{DISCUSSION}

The diffusion coefficient depends on a combination of the static and dynamic properties of the suspended particles, which both are influenced by the direct interactions between these particles. The dependence on dynamic properties stems from the dependence on the mobility which is difficult to evaluate experimentally. Diffusion is a convenient alternative to obtain information about the mobility given the value of the osmotic compressibility. The combined knowledge of the mobility and the osmotic compressibility allows a more detailed knowledge of the interaction potential than each of the individual quantities separately.

\section{ACKNOWLEDGMENTS}

We thank Dr. I. Veretennicoff for several illuminating discussions on this problem. This work has been supported by the Interuniversitair Instituut voor Kernwetenschappen (IIKW).

\section{APPENDIX}

From Eq. (36) we find explicitly for $\delta$,

$$
\delta=-\int_{2 a}^{\infty}\left(\exp \left\{-A \ln \left[1+\exp \left(-\frac{r-2 a}{\xi}\right)\right]\right\}-1\right) d r .
$$

Introducing the new variable

$$
u=A \exp [-(r-2 a) / \xi],
$$

one finds from Eq. (A1),

$$
\delta=\xi \int_{A}^{0}\left[\left(1+\frac{u}{A}\right)^{-A}-1\right] \frac{d u}{u} .
$$

For large values of $A$, the integrand on the right-hand side of Eq. (A2) can be approximated by $\left(e^{-u}-1\right) / u$ and one obtains 


$$
\delta \simeq \xi \int_{0}^{A}\left(1-e^{-u}\right) \frac{d u}{u} .
$$

This leads immediately to

$$
\delta \simeq \xi\left(\ln A+C+\int_{A}^{\infty} e^{-u} \frac{d u}{u}\right),
$$

where we introduced Euler's constant $C$, defined by

$C=\int_{0}^{1}\left(1-e^{-u}\right) \frac{d u}{u}-\int_{1}^{\infty} e^{-u} \frac{d u}{u}=0.5772 \cdots$.

For large values of $A$, the integral

$$
\int_{A}^{\infty} e^{-u} \frac{d u}{u}
$$

is small so that Eq. (A3) reduces to Eq. (40).

${ }^{1}$ J. L. Aguirre and T. J. Murphy, J. Chem. Phys, 59, 1833 (1973).

${ }^{2}$ A. R. Altenberger and J. M. Deutch, J. Chem. Phys. 59, 894 (1973).

${ }^{3}$ A. R. Altenberger, Chem. Phys. 15, 269 (1976).

${ }^{4}$ A. R. Altenberger, J. Chem. Phys. 70, 1994 (1979).

${ }^{5}$ G. D. J. Phillies, J. Chem. Phys. 60, 976 (1974).

${ }^{6}$ G. D. J. Phillies, J. Chem. Phys. 62, 3925 (1975).

${ }^{7}$ J. L. Anderson and C. C. Reed, J. Chem. Phys. 64, 3240 (1975).

${ }^{8}$ W. Hess and R. Klein, Phys. Status Solidi A 85, 509 (1976). ${ }^{9}$ S. Harris, J. Phys. A 9, 1895 (1976).

${ }^{10}$ B. J. Ackerson, J. Chem. Phys. 64, 242 (1976).

${ }^{11}$ B. J. Ackerson, J. Chem. Phys. 69, 689 (1978),

${ }^{12}$ S. A. Allison, E. L. Chang, and J. M. Schurr, Chem. Phys. 38,29 (1979).
${ }^{13}$ G. K. Batchelor, J. Fluid Mech. 74, 1 (1976).

${ }^{14}$ B. U. Felderhof, J. Phys. A 11, 929 (1978).

${ }^{15}$ J. C. Brown, P. N. Pusey, J. W. Goodwin, and R. M. Ottewil, J. Phys. A 8, 664 (1975).

${ }^{16}$ G. D. J. Phillies, G. B. Benedek, and N. A. Mazer, J. Chem. Phys. 65, 1883 (1976).

${ }^{17}$ A. A. Graciaa, J. Lachaise, P. Chabrat, L. Letamendia, J. Rouch, C. Vaucamps, M. Bourrel, and C. Chambu, J. Phys. (Paris) Lett. 38, 253 (1977).

${ }^{18}$ A. A. Graciaa, J. Lachaise, P. Chabrat, L. Letamendia, J. Rouch, and C. Vaucamps, J. Phys. (Paris) Lett. 39, 235 (1978).

${ }^{19}$ B. D. Fair, D. Y. Chao, and A. M. Jamieson, J. Colloid Interface Sci. 66, 323 (1978).

${ }^{20}$ A. M. Cazabat, M. Lagues, D. Langevin, R. Ober, and C. Taupin, C. R. Acad. Sci. Ser. B 287, 25 (1978).

${ }^{21}$ A. M. Cazabat, D. Langevin, and A. Pouchelon, J. Colloid Interface Sci. (to be published).

${ }^{22}$ H. M. Fijnaut, C. Pathmananoharan, E. A. Nieuwenhuis, and A. Vrij, Chem. Phys. Lett. 59, 351 (1978).

${ }^{23}$ A. Einstein, Ann. Phys. (Leipzig) 17, 549 (1905).

${ }^{24}$ A. Einstein, Z. Electrochem. 14, 235 (1908).

${ }^{25}$ L. Onsager, Phys. Rev. 37, 405 (1931).

${ }^{26}$ W. G. McMillan and J. E. Mayer, J. Chem. Phys. 13, 276 (1945).

${ }^{27}$ T. L. Hill, An Introduction to Statistical Mechanics (AddisonWesley, Reading, Mass., 1960), Chap. 19.

${ }^{28}$ J. M. Burgers, Proc. K. Ned. Akad. Wet. 44, 1045, 1177 (1941); $45,9,126$ (1941).

${ }^{29}$ G. K. Batchelor, J. Fluid Mech. 52, 245 (1972).

${ }^{30}$ M. Stimson and G. B. Jeffery, Proc. R. Soc. London Ser. A 111,110 (1926).

${ }^{31}$ A. J. Goldman, R. G. Cox, and H. Brenner, Chem. Eng. Sci. 21, 1151 (1966).

${ }^{32}$ G. D. J. Phillies, J. Chem. Phys. 67, 4690 (1977).

${ }^{33} \mathrm{E}$. J. W. Verwey and. J. Th. G. Overbeek, Theory of the Stability of Lyophobic Colloids (Elsevier, Amsterdam, 1948).

${ }^{34}$ L. Onsager, Ann. N. Y. Acad. Sci. 51, 627 (1949). 